\title{
Capture of airborne nanoparticles in swirling flows using non-uniform electrostatic fields for bio- sensor applications
}

Jaesung Jang

Laboratory of Integrated Biomedical Micro/Nanotechnology and Applications, Birck Nanotechnology Center, Bindley Biosciences Center, School of Electrical and Computer Engineering, Purdue University

Demir Akin

Purdue University, demirakin@gmail.com

Kwan Seop Lim

Birck Nanotechnology Center, Bindley Bioscience Center, School of Electrical and Computer Engineering, Weldon School of Biomedical Engineering, Purdue University; ERC for Advanced Bioseparation Technology, Inha University, limk@purdue.edu

Steve Broyles

Department of Biochemistry, Purdue University, broyles@purdue.edu

Michael R. Ladisch

Weldon School of Biomedical Engineering, Purdue University, ladisch@purdue.edu

See next page for additional authors

Follow this and additional works at: http://docs.lib.purdue.edu/nanodocs

Jang, Jaesung; Akin, Demir; Lim, Kwan Seop; Broyles, Steve; Ladisch, Michael R.; and Bashir, Rashid, "Capture of airborne nanoparticles in swirling flows using non-uniform electrostatic fields for bio-sensor applications" (2006). Other Nanotechnology Publications. Paper 3.

http://docs.lib.purdue.edu/nanodocs/3 
Authors

Jaesung Jang, Demir Akin, Kwan Seop Lim, Steve Broyles, Michael R. Ladisch, and Rashid Bashir 


\title{
Capture of airborne nanoparticles in swirling flows using non-uniform electrostatic fields for bio-sensor applications
}

\author{
Jaesung Jang a , Demir Akin ${ }^{a}$, Kwan Seop Lim ${ }^{\mathrm{a}, \mathrm{e}}$, Steven Broyles ${ }^{\mathrm{d}}$, \\ Michael R. Ladisch ${ }^{\mathrm{b}, \mathrm{c}}$, Rashid Bashir ${ }^{\mathrm{a}, \mathrm{c}, *}$

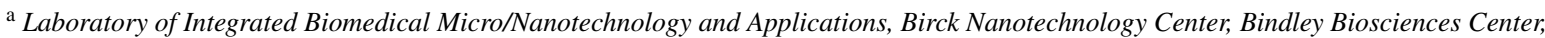 \\ School of Electrical and Computer Engineering, Purdue University, West Lafayette, IN 47907, United States \\ ${ }^{\mathrm{b}}$ Laboratory of Renewable Resources Engineering (LORRE), Department of Agricultural and Biological Engineering, Purdue University, \\ West Lafayette, IN 47907, United States \\ ${ }^{\mathrm{c}}$ Weldon School of Biomedical Engineering, Purdue University, West Lafayette, IN 47907, United States \\ d Department of Biochemistry, Purdue University, West Lafayette, IN 47907, United States \\ e Engineering Research Center for Advanced Bioseparation Technology, Inha University, Incheon 402-751, Korea
}

Received 6 January 2006; accepted 20 April 2006

Available online 2 June 2006

\begin{abstract}
Collection of biological particles is the first and critical step for any biological agent detection system. Towards our goal of capturing and detecting airborne biological entities in real time, here we investigate on the design of an electrostatic particle capture system. We report on the capture of airborne $100 \mathrm{~nm}$ diameter polystyrene nanoparticles as a model system, in swirling flows under non-uniform electrostatic fields with an electrospray aerosol generator and a homemade particle collector. The particle collector has five positive electrodes on the bottom and one large grounded electrode on the top. The nanoparticles coming into the collector were slowed down during their swirling and stayed in the collector long before leaving the collector. Silicon chips were placed on the bottom electrodes and the electrostatically captured particles were counted as a function of flow rates, electrode positions, bias voltages, and capture times by epifluorescent images and scanning electron micrographs (SEMs). Particles captured in the electrode at the center of the collector were much less than those on the surrounding four electrodes and 10-25\% of the particles with negative charges entering the collector were captured on the bottom electrodes at a flow rate of $1.11 / \mathrm{min}$ and an applied potential of $2 \mathrm{kV}$. Particle capture increased with decreasing flow rates. We also simulated flow and electrical fields separately, and found the positional trends to be in good agreement with the measurements. This collector is well adaptable to integration with micro resonator devices and can be used for real-time monitoring of bioaerosols.
\end{abstract}

(c) 2006 Elsevier B.V. All rights reserved.

Keywords: Airborne nanoparticles; Electrostatic capture; Swirling flows

\section{Introduction}

Recently, collection of biological aerosols, airborne bacteria or viruses, has received significant interest for a wide variety of applications such as healthcare, industrial microbiology, bioterrorism, etc. [1-4]. Exposure to bioaerosols in indoor and outdoor environments may cause a wide range of health disorders, ranging from mild irritation to a serious disease. Typically bioaerosols are collected and then examined in the gas phase

\footnotetext{
* Corresponding author. Tel.: +1 7654966229.

E-mail address: bashir@purdue.edu (R. Bashir).
}

or most often introduced into a liquid medium, where they can be further characterized to determine the presence of specific biological targets [4].

Electrostatic precipitators or collectors have been commonly used to capture or control airborne dust and fine particles in indoor air cleaning systems and industry [5]. Various electrostatic collectors have also been developed to capture bioaerosols from airstreams [1-3]. These instruments have an advantage of being operated with a low-pressure drop. They use corona dischargers to charge incoming aerosols and collect these charged aerosols by electrostatic forces. They have shown high collection efficiencies that can reach almost $99 \%$ due to high electrical mobility of those multiply charged particles [5]. However, 
corona dischargers are well known to produce oxides of nitrogen and ozone, which may adversely affect the viability of some airborne microorganisms [6,7].

In this work, we present an airborne particle collector using non-uniform electrostatic fields and swirling gas flows without a corona discharger. We used polystyrene nanoparticles as the model entities to perform the characterization. Flow swirling makes the particles stay longer in the collector and increases the probability of electrostatic capture. To show the effects of flow fields on the collection efficiency, we also present flow and electrical simulations in the collector using commercial computing softwares. During the flow of the nanoparticles in the collector, the particles are slowed down and hence the probability of capture is increased. We also present particle counts of collected airborne nanoparticles and those variations with position in the collector for different operating conditions. The nanoparticles are collected on smaller areas of chips, increasing the particle number density. This collector is ideally suited for integration with micro resonator devices operating in gaseous state, which provide higher sensitivities than in the liquid state due to lower viscous damping. These collectors can be promising candidates as real-time monitoring devices, which can be highly beneficial due to their short response times for a wide range of applications $[4,8,9]$.

\section{Experimental setup}

Fig. 1 shows a schematic of the particle generation and collection system, and the top and side views of the collector.

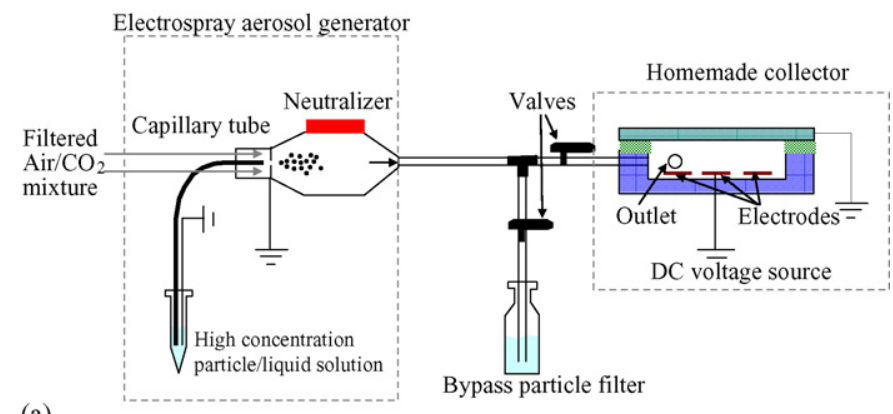

(a)

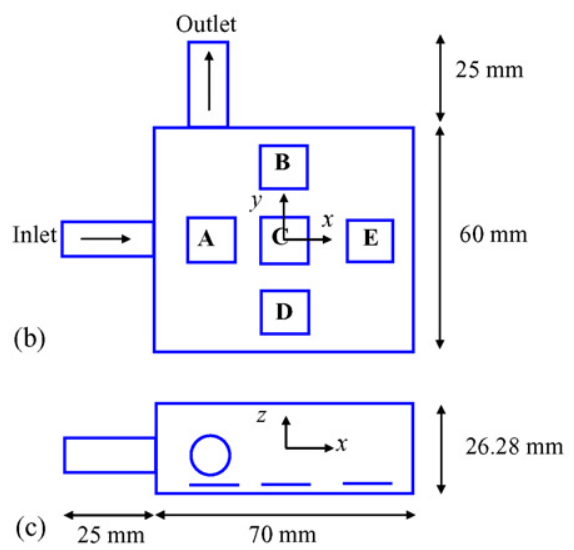

Fig. 1. Schematic of a particle generation and collection system (a), the top view of the collector (b), and the side view (c). The capitals in (b) indicate each electrode.
The collector is $70 \mathrm{~mm}$ long, $60 \mathrm{~mm}$ wide, and $26.28 \mathrm{~mm}$ high. Five metal sheets $(12 \mathrm{~mm} \times 12 \mathrm{~mm})$ were affixed to different positions at the bottom of the collector, acting as the bottom electrodes to capture the airborne nanoparticles. The nanoparticles used were $100 \mathrm{~nm}$ diameter polystyrene fluorescent beads (R100, Duke Scientific Corp., Palo Alto, CA, USA) and the particle concentration in the solution was $10^{11} / \mathrm{ml}$. The sheath air was cleaned with a filtered air supply (Model 3074B, TSI Inc., St. Paul, MN, USA) and $\mathrm{CO}_{2}$ was supplied from a gas cylinder. The nanoparticles in the solution were passed through the capillary tube and the monodisperse aerosolized particles were formed using an electrospray aerosol generator (Model 3480, TSI Inc., St. Paul, MN, USA). The generated aerosols were neutralized by a Polonium-210 source (Po-2042, NRD Inc., Grand Island, NY, USA), resulting in the nanoparticles in charge equilibrium with about one third of them having one or more negative charges [10]. The nanoparticles pass through a tube whose length between the exit of the aerosol generator and the inlet of the collector is about $40 \mathrm{~cm}$. This tube was conductive and grounded to minimize electrostatic losses of particles. The aerosols leaving the collector were passed through a HEPA filter before going to the environment.

A silicon chip $(10 \mathrm{~mm} \times 10 \mathrm{~mm})$ was placed on one of the bottom electrodes for the capture experiments. As the particle generation gets stabilized, the valve to the collector was opened and the bypass valve was closed. Electric potential was applied on the bottom electrode for either 5 or $20 \mathrm{~min}$ while the top electrode was grounded. The applied potential ranged from 0 to $2 \mathrm{kV}$ and was applied using a dc power supply. Five different electrode positions inside the collector were investigated to examine the captured particle counts versus the position in the collector. The captured particle counts on the silicon chips were also examined versus flow rates. The nanoparticles captured on the silicon chip were imaged under an epifluorescent microscope and a scanning electron microscope. In order to find the number of particles entering the collector and particle losses along the tubes before the inlet of the collector, the aerosols entering the collector were captured into deionized water and were counted with a fluorometer (Cary Eclipse, Varian Inc., Palo Alto, CA, USA).

\section{Measurements of particle counts}

Fig. 2 shows the fluorescent images $(217.75 \mu \mathrm{m} \times$ $172.53 \mu \mathrm{m})$ taken from the silicon chips placed at five different electrode locations in the collector where the electric potential of $2 \mathrm{kV}$ was applied for $20 \mathrm{~min}$ at a gas mixture flow rate of $1.1 \mathrm{l} / \mathrm{min}$, where the $\mathrm{CO}_{2}$ flow rate was $0.1 \mathrm{l} / \mathrm{min}$. The particles in all the images are well dispersed and their aggregation is so rare that we can identify almost all the single particles. The particles in these pictures were counted with ImageJ, a free software distributed by the US National Institute of Health. The scanning electron micrographs (SEMs) of these silicon chips were also taken and the number of particles from the SEMs were counted. Table 1 shows the number of particles counted by the SEMs and fluorescent images in an area of $36.6 \mu \mathrm{m} \times 36.6 \mu \mathrm{m}$. The values in the parentheses are standard deviations of the measurements. 
(A)

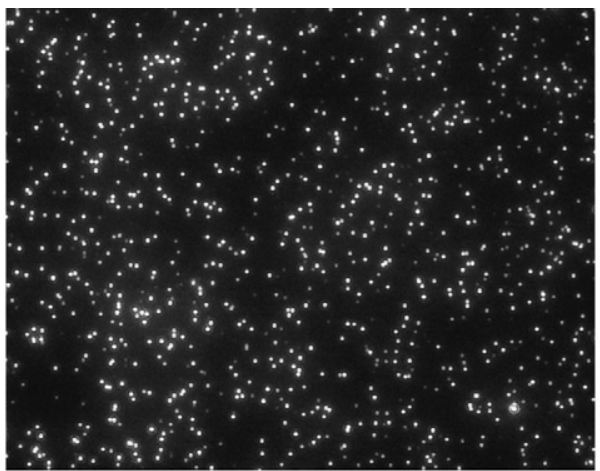

(C)

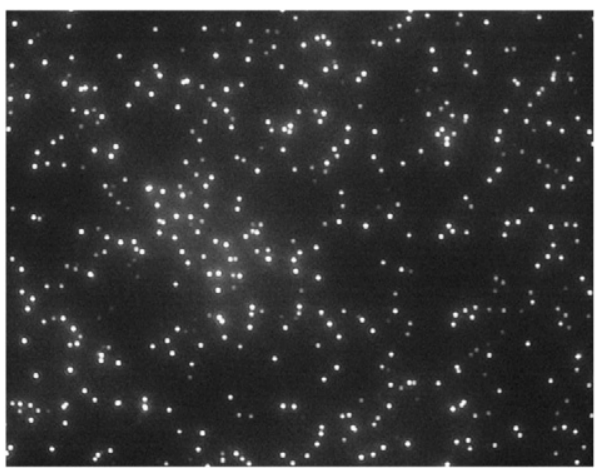

(B)

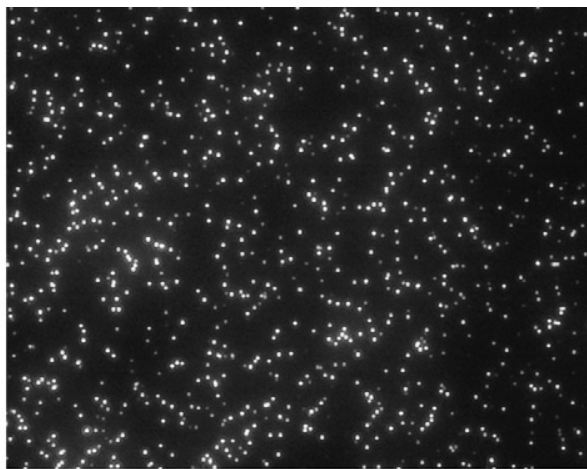

(D)

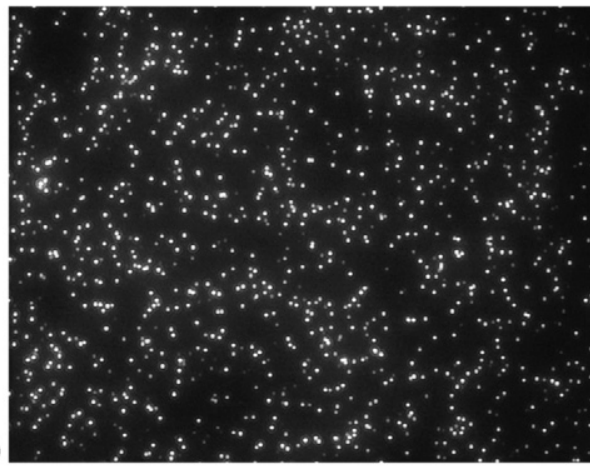

(E)

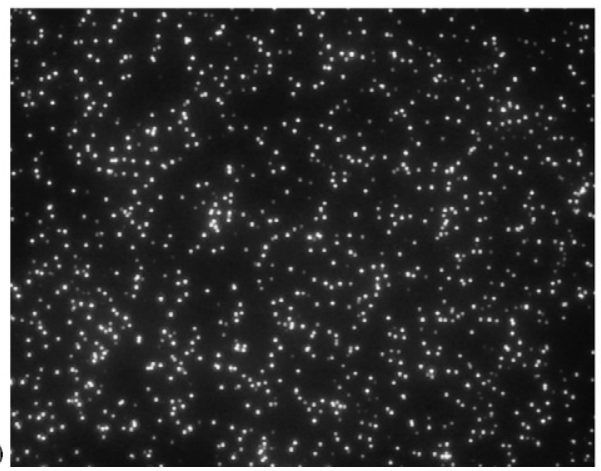

Fig. 2. Fluorescent images $(217.75 \mu \mathrm{m} \times 172.53 \mu \mathrm{m})$ taken from the silicon chips placed at five different electrodes in the collector where the electric potential of $2 \mathrm{kV}$ was applied for $20 \mathrm{~min}$ at a flow rate of $1.1 \mathrm{l} / \mathrm{min}$. The capitals in the parentheses indicate each electrode.

The counts by both methods are overlapped within their standard deviations except for the electrode A, which may be attributed to bad sampling of the SEMs. In fact, it is observed that the standard deviations are larger in the SEMs. In both methods, the particle counts in the electrodes A, B, D, and E are shown to be close within their uncertainties, while the particle counts captured in the electrode $\mathrm{C}$ are much less than those in the other four electrodes.

\section{Table 1}

Number of particles in an area of $36.6 \mu \mathrm{m} \times 36.6 \mu \mathrm{m}$ counted by SEMs and fluorescent images, where the values in the parentheses are standard deviations

\begin{tabular}{llllll}
\hline & \multicolumn{4}{l}{ Electrode area } & \\
\cline { 2 - 6 } & $\mathrm{A}$ & $\mathrm{B}$ & $\mathrm{C}$ & $\mathrm{D}$ & $\mathrm{E}$ \\
\hline SEM & $38.5(3.5)$ & $26.3(5.9)$ & $15.0(8.2)$ & $37.3(7.0)$ & $39.3(11.4)$ \\
Fluorescent & $29.2(1.1)$ & $27.4(1.7)$ & $14.6(0.5)$ & $32.0(3.0)$ & $34.2(1.4)$ \\
\hline
\end{tabular}

Fig. 3 shows the total number of particles captured in an area of $217.8 \mu \mathrm{m} \times 172.5 \mu \mathrm{m}$ as a function of the different applied voltage (a) and time of capture (b) for the different positions, where the error bars represent standard deviations of the measurements. In (a), the electric potential was applied for $20 \mathrm{~min}$ at a gas mixture flow rate of $1.1 \mathrm{l} / \mathrm{min}$ and in (b), the electric potential of $2 \mathrm{kV}$ was applied at a gas mixture flow rate of $1.1 \mathrm{l} / \mathrm{min}$. The captured particle counts increased with increasing applied electrical potential and time. In this figure, it can also be observed that the captured particle counts on the electrode $\mathrm{C}$ is much less than those in the electrodes A, B, D, and E. Fig. 4 shows the collection efficiencies versus flow rates of a gas mixture of air/ $\mathrm{CO}_{2}$ when the electric potential of $2 \mathrm{kV}$ was applied for $20 \mathrm{~min}$. The collection efficiency is defined as the ratio of the total number of particles captured on the electrode to the total number of particles coming into the collector. The collection efficiency increased with the decreasing flow rates and it was $7-8 \%$ at a flow rate of $1.1 \mathrm{l} / \mathrm{min}$ on the electrodes A, B, D, and E. This means that 


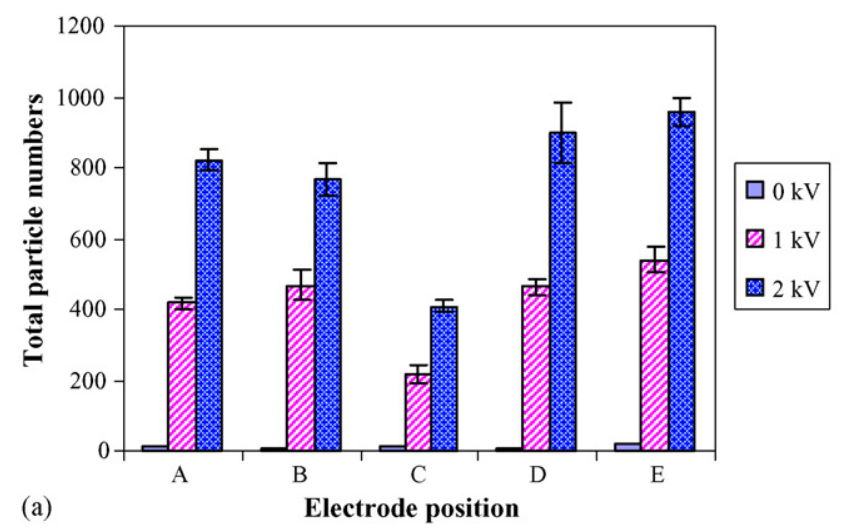

(a)

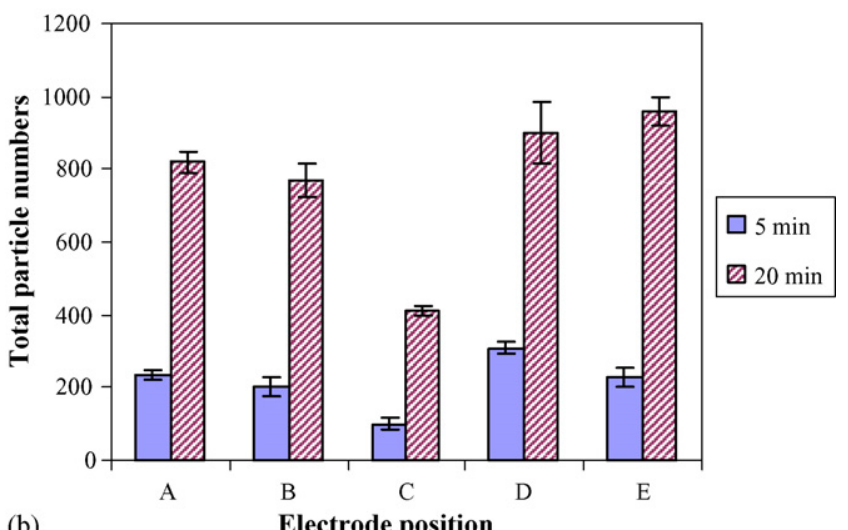

(b)

Fig. 3. Total number of particles captured in an area of $217.8 \mu \mathrm{m} \times 172.5 \mu \mathrm{m}$ with applying voltage (a) and applying time (b), where the error bars represent standard deviations of the measurements.

about $20-25 \%$ of the particles having negative charges can be captured because $34 \%$ of the total particles entering the collector are known to have negative charges and should be attracted to the positive bottom electrodes. The particle losses were also measured while they flow from the capillary tip to the inlet of the collector by comparing the total number of particles generated from the capillary tip with the measured particle counts entering the collector. The total number of particles generated from the capillary tip can be calculated by multiplying the liquid flow rate of the solution along the capillary tube by the particle

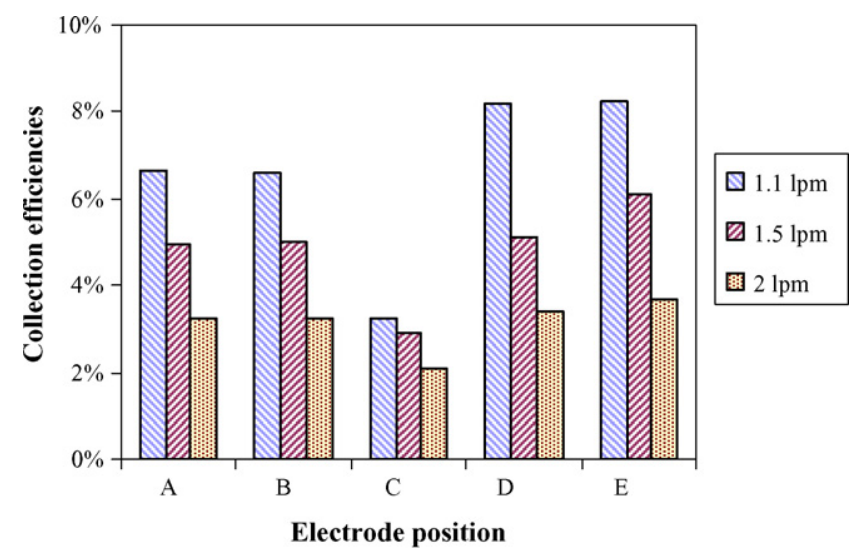

Fig. 4. Collection efficiencies vs. flow rates of a gas mixture of air/ $/ \mathrm{CO}_{2}$ with electrode positions when the electric potential of $2 \mathrm{kV}$ was applied for $20 \mathrm{~min}$. concentration in the liquid solution and capture time. The particles entering the collector were counted by capturing them into deionized water and then measuring their fluorescence. The fluorescence signal was calibrated by using control solutions whose particle concentration ranged from $10^{6}$ to $10^{9} / \mathrm{ml}$ and the optical output was found to be linear with particle concentration. It was found that $\sim 9.5 \%$ of the total particles generated from the fluid source entered the collector. This loss of efficiency may be due to particle losses caused by flow constrictions through valves, tube connections such as a tee, and the neutralizer.

\section{Simulation and comparison with measurements}

Airborne particles under non-uniform electrostatic fields experience electrical forces, flow-induced forces, gravity, etc. [11]. Electrical forces include electrophoretic forces due to electrostatic fields and dielectrophoretic forces due to nonuniformity of the electric fields. Flow induced forces include viscous drag and lift forces. Dielectrophoretic forces are limited to only an area near the electrodes and can be negligible compared to electrophoretic forces in most regions of the collector. Therefore, the movements of airborne particles are mostly influenced by electrophoretic forces and interaction with fluid flows [12]. That is, electric and flow fields are required in the particle collector design. Generally, the collector should be designed such that particles move slow enough to be captured with given electric field intensity in order to maximize their collection.

Flow and electric fields were obtained separately and particles were traced from flow fields without considering the effects of electric fields on the particles. These particle tracks still give important information on the relationship between particle velocities and the captured particle counts on the electrodes by electrophoretic forces. Electrophoretic forces exerted on a charged particle are given by [11]

$\vec{F}_{\mathrm{E}}=n e \vec{E}$

where $n$ is the number of elementary charge units on a particle, $e$ the elementary unit of charge $\left(=1.602 \times 10^{-19} \mathrm{C}\right)$, and $\vec{E}$ is the electric field intensity. According to the equilibrium charge distribution produced by the neutralizer, about $28 \%$ of the total particles $100 \mathrm{~nm}$ in diameter have single negative charges $(n=-1)$ and $6 \%$ of the total particles have multiple negative charges [10]. If a particle is placed in an electric field, it will reach a settling velocity, which is given by [11]

$\vec{v}_{\mathrm{p}}=\frac{n e C_{\text {slip }}}{6 \pi a \eta} \vec{E}$

where $\eta$ is the dynamic viscosity of the fluid, $a$ the radius of a particle, and

$C_{\text {slip }}=1+\frac{1}{p d_{\mathrm{p}}}\left[15.60+7.00 \exp \left(-0.059 p d_{\mathrm{p}}\right)\right]$

is the slip correction factor, where $p$ is the absolute pressure in $\mathrm{kPa}$ and $d_{\mathrm{p}}$ is the particle diameter in $\mu \mathrm{m}$ [11].

Particle traces in the collector were numerically computed with a commercial finite volume CFD code FLUENT 6.2. Gravity and interaction with surrounding flows were considered and 
Table 2

Material properties of the particles and the operating conditions used in flow and electrical simulations at a gas flow rate of $1.1 \mathrm{l} / \mathrm{min}$

\begin{tabular}{ll}
\hline Polystyrene diameter $(\mathrm{nm})$ & 100 \\
Polystyrene relative permittivity & 2.6 \\
Polystyrene density $\left(\mathrm{kg} / \mathrm{m}^{3}\right)$ & 1050 \\
Temperature $(\mathrm{K})$ & 300 \\
Ambient pressure $(\mathrm{kPa})$ & 101.3 \\
Slip correction factor & 2.92 \\
Reynolds number based on the inlet diameter & 137 \\
Applying voltage difference $(\mathrm{kV})$ & 2.0 \\
\hline
\end{tabular}

flow fields were obtained based on the incompressible laminar flow model. Table 2 shows the material properties of the particles and the operating conditions used in the flow and electrical simulations, where the electric potential of $2 \mathrm{kV}$ was applied at a gas mixture flow rate of $1.1 / \mathrm{min}$. Fig. 5 shows the positions of 69 test particles starting on the inlet surface and a representative particle trajectory. The test particles are equally spaced on the inlet surface. Fig. 6 shows the velocity magnitudes of the test particles with time while they flow from the inlet to the outlet. All the test particles experience reduced velocities when they enter larger spaces inside the collector. Their velocities increase again when they leave the collector. It should be noted that not all the particles leave the collector at the same time. Some particles go to the outlet directly without swirling and some particles
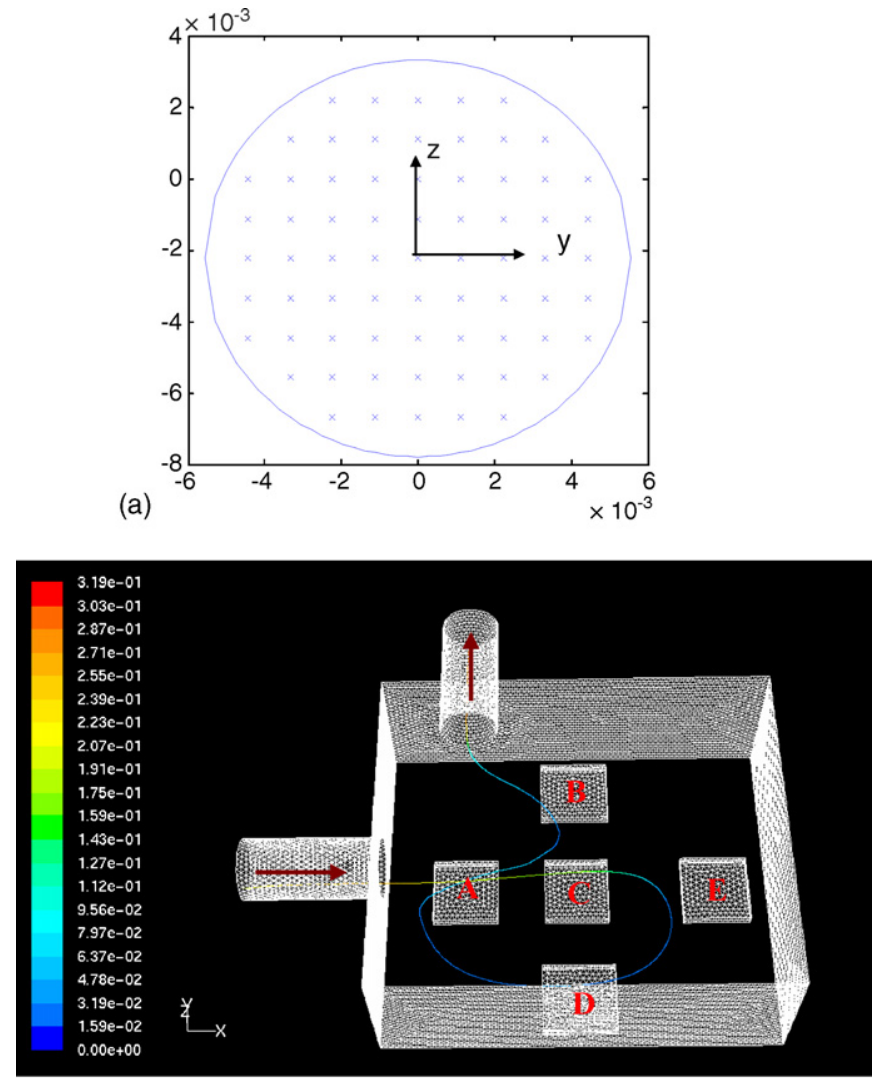

(b)

Fig. 5. Positions of 69 test particles starting at the inlet surface (a) and a representative particle trajectory at an inlet Reynolds number of 137 (b).
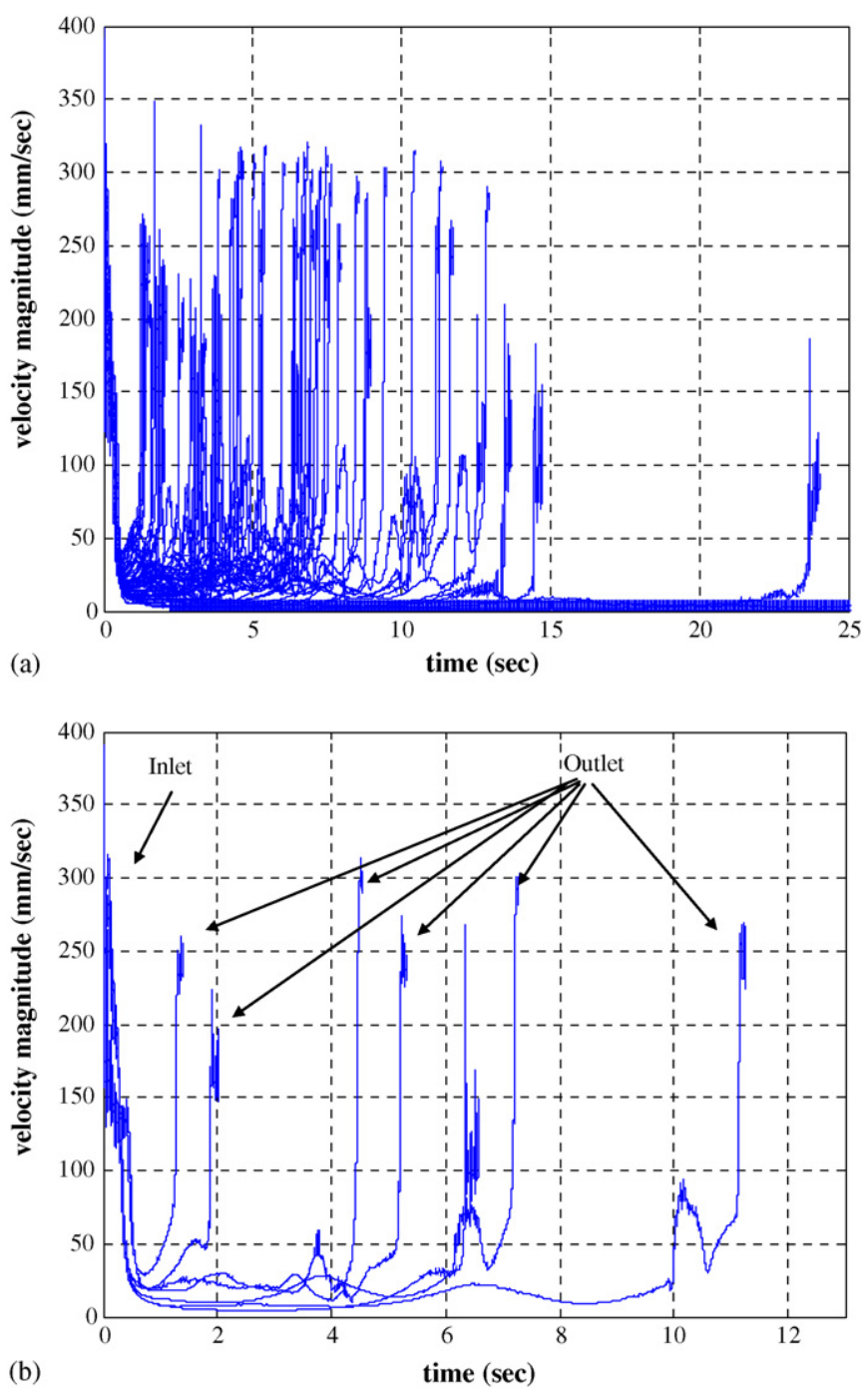

Fig. 6. Velocity magnitudes of 69 particles (a) and 7 particles (b) out of the 69 particles with time while they flow from the inlet to the outlet.

stay much longer while swirling in the collector, making it more probable for them to be captured.

Table 3 shows the number of particles passing through the volumes made up of the electrode areas and collector height, and the expanded volumes comprised of the heights and expanded areas which have each edge of the electrode extended by $4 \mathrm{~mm}$. These expanded volumes may be more appropriate to examine around electrodes $\mathrm{D}$ and $\mathrm{E}$ because there are more slow particles around these electrodes. In fact, these slow particles can be affected by non-uniform electric fields even when they are not right over the electrodes.

Fig. 7 shows the minimum horizontal velocities that all the particles can achieve when passing through the expanded volumes versus the distance of the particles from each electrode. There are three different regions in this figure. One of them is where particles have high velocities close to the electrodes or low velocities far away from the electrodes. Another region is where particles are moving slow near the electrodes and electric field intensity is large. The particles in this region are slow enough to be collected on the electrode, so this is where most of 
Table 3

Number of particles passing through volumes made up of the electrode areas and collector heights and the expanded volumes being comprised of the heights and expanded areas which have each edge of the electrode extended by $4 \mathrm{~mm}$

\begin{tabular}{llllll}
\hline & Electrode & & & \\
\cline { 2 - 5 } & A & B & C & D & E \\
\hline Expanded volume & $69(100 \%)$ & $60(87 \%)$ & $69(100 \%)$ & $28(41 \%)$ & $22(31 \%)$ \\
Electrode volume only & $69(100 \%)$ & $46(67 \%)$ & $61(88 \%)$ & $12(17 \%)$ \\
\hline
\end{tabular}

the particle collection on the electrodes happens. On the other hand, in the region where particles are fast and distant from the electrodes, electrostatic forces are not large enough to change the directions of the particles to the electrodes due to their high momentum. This can explain why the electrodes $\mathrm{A}$ and $\mathrm{C}$ have fewer particles captured in the measurements than the actual numbers of particles passing over the electrodes as shown in Table 3. That is, there are many particles right over the electrode $\mathrm{C}$, but most of them have high velocities. On the other hand, there are small numbers of particles over the electrodes D and E, but most of them are moving slow enough to be captured. According to the results above, it can be concluded that the electrodes $\mathrm{D}$ or $\mathrm{E}$ is a better place for microresonator chips to collect bioaerosols and the electrode $\mathrm{C}$ is not appropriate unless the aerosols are highly charged.

Electric fields are computed with a finite element software ANSYS 9.0. Fig. 8 shows maximum vertical settling velocities of particles having single negative charges due to electrophoretic forces at each height from the electrodes. The settling velocities of particles increase as they move closer to the electrode. It is shown that the effects of the electric fields on the particles are almost the same all over the electrodes, so the differences of the captured particle counts with the electrode positions can be mostly determined by particles' tracks and their velocities. It should be noted that this maximum vertical settling velocity is a measure of particle capturing. In fact, these velocities do not

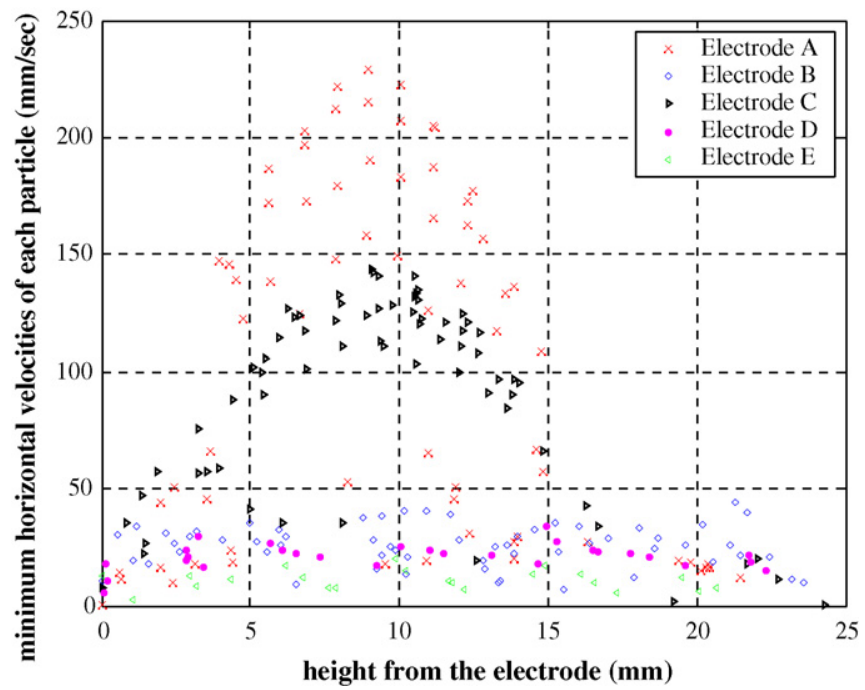

Fig. 7. Minimum horizontal velocities that all the particles can get when passing through the expanded volumes vs. the distance of the particles from each electrode.

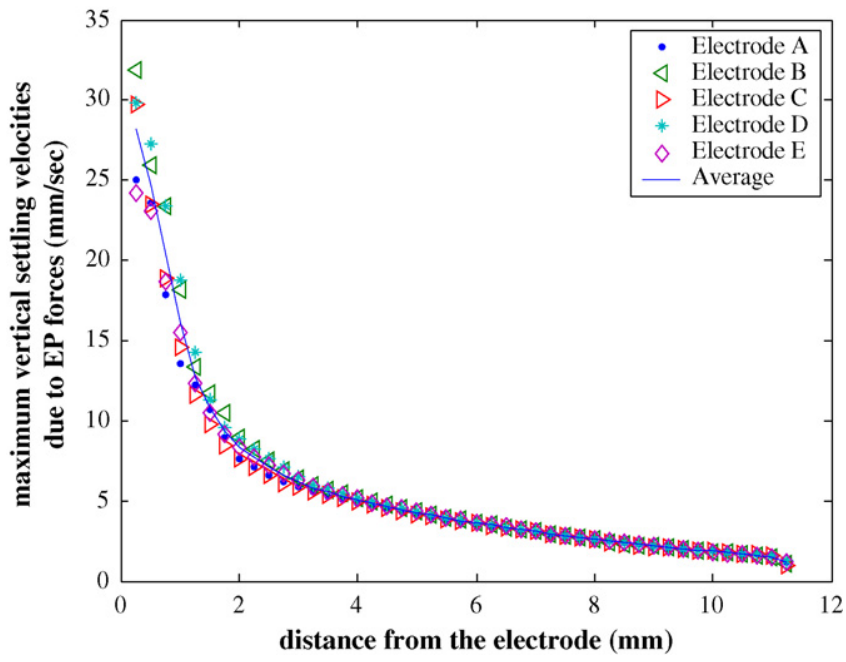

Fig. 8. Maximum vertical settling velocities of particles having single negative charges due to electrophoretic forces at each height from the electrodes.

directly relate to the critical velocities of particles in order to be captured, since the electric fields are not constant with height, so even faster particles than these velocities can be captured if they are moved closer to the electrodes, which produces larger electric forces on the particles.

\section{Conclusions}

We presented a homemade particle collector to capture $100 \mathrm{~nm}$ diameter airborne polystyrene nanoparticles in swirling gas flows using non uniform electrostatic fields. The collector is $70 \mathrm{~mm}$ long, $60 \mathrm{~mm}$ wide, and $26.28 \mathrm{~mm}$ high and has five bottom electrodes and a large top electrode. We also characterized and presented the nanoparticle capture with flow rate, applied voltage, electrode position, and time. The captured particle counts on the electrodes A, B, D, and E were shown to be close within their uncertainties, while the particles captured in the electrode $\mathrm{C}$ were much less than those in the other four electrodes. Only $20-25 \%$ of the negatively charged particles entering the collector were captured on the electrodes (except for the center position) at a flow rate of $1.1 \mathrm{l} / \mathrm{min}$ and an electric field of $2 \mathrm{kV}$. It was also determined that $\sim 9.5 \%$ of the total particles generated at the capillary tip entered the collector.

We simulated flow and electrical fields in the collector separately. All the test particles entered the collector at reduced velocities. Some particles went to the outlet directly, but most particles stayed much longer before leaving the collector. There were many particles passing right over the electrode $\mathrm{C}$, but most 
of them were too fast to be captured. On the other hand, there were small numbers of particles passing over the electrodes D and $\mathrm{E}$, but most of them were moving slow and hence were captured. This explains why the electrode $\mathrm{C}$ had fewer particles captured in the measurements. The electrodes D or E presented a better place for placement of sensor elements such as microresonator chips to collect aerosols, as compared to the electrode C. We are continuing to expand this work and are integrating such sensor elements for real time capture and detection of air-borne virus particles.

\section{Acknowledgements}

The authors thank Dr. Tom Huang, Dr. Amit Gupta, and Mr. Himadri Pal for their valuable discussions and Prof. Donald E. Bergstrom for fluorescence measurements. We are also thankful for the financial support of the US National Institute of Health (NIBIB grant number R21/R33 EB00778-01) for funding Dr. Jaesung Jang and Dr. Demir Akin. We would also like to acknowledge the group of Dr. Debby Sherman for assistance with SEM imaging. Dr. Kwan Seop Lim was supported by the Engineering Research Center for the Advanced Bioseparation Technology, KOSEF, Korea, during his stay at Purdue University.

\section{References}

[1] G. Mainelis, K. Willeke, A. Adhikari, T. Reponen, S.A. Grinshpun, Design and collection efficiency of a new electrostatic precipitator for bioaerosol collection, Aerosol Sci. Technol. 36 (2002) 1073-1085.

[2] C.-S. Li, Y.-M. Wen, Control effectiveness of electrostatic precipitation on airborne microorganisms, Aerosol Sci. Technol. 37 (2003) 933-938.

[3] C.J. Horgan Jr., M.-H. Lee, P. Biswas, Capture of viral particles in soft $\mathrm{X}$-ray-enhanced corona systems: charge distribution and transport characteristics, Aerosol Sci. Technol. 38 (2004) 475-486.

[4] A.A. Fatah, J.A. Barrett, R.D. Arcilesi Jr., K.J. Ewing, C.H. Lattin, LTC.T.F. Moshier, An Introduction to Biological Agent Detection Equipment for Emergency First Responders: NIJ Guide 101-00, National Institute of Justice, 2001.

[5] A. Mizuno, Electrostatic precipitation, IEEE Trans. Dielectr. Electr. Insul. 7 (2000) 615-624.

[6] B. Crook, Non-inertial samplers: biological perspectives, in: C.S. Cox, C.M. Wathes (Eds.), Bioaerosols Handbook, Lewis Publishers, 1995.

[7] W.J. Kowalski, W.P. Bahnfleth, B.A. Striebig, T.S. Whittam, Demonstration of a hermetic airborne ozone disinfection system: studies on E. coli, Am. Ind. Hyg. Assoc. J. 64 (2003) 222-227.

[8] A. Gupta, D. Akin, R. Bashir, Single virus particle mass detection using microresonators with nanoscale thickness, Appl. Phys. Lett. 84 (2004) 1976-1978.

[9] R.L. Gunter, W.G. Delinger, K. Manygoats, A. Kooser, T.L. Porter, Viral detection using an embedded piezoresistive microcantilever sensor, Sens. Actuator, A Phys. 107 (2003) 219-224.

[10] A. Wiedensohler, Technical note: an approximation of the bipolar charge distribution for particles in the submicron size range, J. Aerosol Sci. 19 (1988) 387-389.

[11] P.A. Baron, K. Willeke, Gas and particle motion, in: P.A. Baron, K. Willeke (Eds.), Aerosol Measurement: Principles, Techniques, and Applications, second ed., John Wiley \& Sons Inc., 2001.

[12] J. Dixkens, H. Fissan, Development of an electrostatic precipitator for offline particle analysis, Aerosol Sci. Technol. 30 (1999) 438-453.

\section{Biographies}

Jaesung Jang received his B.S. and M.S. degrees in mechanical engineering from Pohang University of Science and Technology (POSTECH), Korea, in 1997 and 1999, respectively, and the Ph.D. degree in mechanical engineering from Purdue University, West Lafayette, IN, in 2004. He is currently working as a post doctoral research associate in electrical and computer engineering at Purdue University, West Lafayette, IN, developing nanomechanical resonant cantilever sensors to detect airborne viruses. His current research interests include fundamental microscale gas and liquid flow characteristics and microelectromechanical sensors and actuators for biomedical and microfluidic systems. He is a member of the American Society of Mechanical Engineers (ASME).

Demir Akin received the D.V.M. degree from Ankara University, Turkey, in 1988, the M.S. degree in microbiology from Mississippi State University in 1991, and the Ph.D. degree in molecular virology from Purdue University,West Lafayette, IN, in 1998. He did his postdoctoral work in the areas of molecular biology and viral bioinformatics at the Indiana State Animal Disease Diagnostic Laboratory from 1998 to 2000. He became a Research Scientist in the School of Nuclear Engineering at Purdue University in 2001 and worked on artificial intelligence-based In-Silico Biology and Genomics software development. As a research scientist, he joined the Electrical and Computer Engineering Department at Purdue University in 2002 and became a Senior Research Scientist in 2003. His current research projects and interests include integration of biology in engineering, nanomedicine, BioMEMS-based sensors and devices with medical diagnostic and therapeutic potential, single molecule imaging using fluorescence microscopy and atomic force microscopy, systems biology, and pathogenic and biodefense-related infectious agent diagnostics.

Kwan Seop Lim received his Ph.D. in analytical chemistry from Pohang University of Science and Technology, South Korea in 2003. He is working as a postdoctoral research scientist in Center for Advanced Bioseparation Technology, Inha University, South Korea and also a visiting scholar in School of Electrical and Computer Engineering, Purdue University, USA. His current researches are focused on developing lab-on-a-chip based bioanalytical system and its applications.

Michael R. Ladisch is Director of the Laboratory of Renewable Resources Engineering and Distinguished Professor of Agricultural and Biological Engineering and Biomedical Engineering at Purdue University. He earned his B.S. from Drexel University and M.S. and Ph.D. degrees from Purdue University, all in chemical engineering. He is currently investigating the scale-down of bioseparations and the rapid prototyping of microfluidic biochips for the rapid detection of pathogenic microorganisms as part of an interdisciplinary team that is addressing the rapid detection of food pathogens. He is a member of National Academy of Engineering.

Rashid Bashir completed his Ph.D. from Purdue University in 1992. From Oct 1992 to Oct 1998, he worked at National Semiconductor in the Process Technology Development Group as Sr. Engineering Manager. He is currently Professor of Electrical and Computer Engineering and Courtesy Professor of Biomedical Engineering at Purdue University. He has authored or coauthored over 100 journal and conference papers and has over 25 patents. His research interests include biomedical microelectromechanical systems, applications of semiconductor fabrication to biomedical engineering, advanced semiconductor fabrication techniques, and nano-biotechnology. In 2000, he received the NSF Career Award for his work in Biosensors and BioMEMS. He received the Joel and Spira Outstanding Teaching award from School of ECE at Purdue University. He was selected by National Academy of Engineering to attend the Frontiers in Engineering Workshop in Fall 2003. He was also a finalist in the Small Times Magazine 2005 Innovator of the Year Award. He is a Senior member of IEEE. 